\title{
Optical design for MEGARA: a multi-object spectrograph for the GTC
}

\author{
Esperanza Carrasco $^{\mathrm{a}}$, Ernesto Sánchez-Blanco ${ }^{\mathrm{b}}$, María Luisa García Vargas ${ }^{\mathrm{b}}$, Armando Gil de Paz ${ }^{\mathrm{c}}$, \\ Jesús Gallego $^{\mathrm{c}}$, Gonzalo Páez ${ }^{\mathrm{d}}$, Jaime Zamorano ${ }^{\mathrm{c}}$ \& Jorge Castro ${ }^{\mathrm{a}}$ \\ ${ }^{a}$ Instituto Nacional de Astrofísica, Óptica y Electrónica, Luis Enrique Erro 1, Tonantzintla, Puebla, \\ Mexico CP 72840; \\ ${ }^{\mathrm{b}}$ Fractal SLNE, C/Tulipán 2, P13-1A, Las Rozas de Madrid, Spain E-28231; \\ ${ }^{\mathrm{c}}$ GUAIX group, Dept. Astrophysics, Univ. Complutense, Avda. Complutense \\ s/n, 28040 Madrid; \\ ${ }^{\mathrm{d}}$ Centro de Investigaciones en Óptica, Loma del Bosque 115, León, Gto., Mexico CP 37154
}

\begin{abstract}
MEGARA is a multi-object spectrograph project for the $10.4 \mathrm{~m}$ Gran Telescopio Canarias with medium to high resolution: $\mathrm{R} \sim 5600-17000$. The instrument operates in three modes that cover different sky areas and that can run simultaneously: (1) the compact mode through a large central Integral Field Unit with minimum fiber pitch, covering a field of view on sky of $12 \operatorname{arcsec} \times 14 \operatorname{arcsec}$, (2) the sparse mode with fibers covering 1 arcmin x 1 arcmin in three pointings and (3) the dispersed mode with a grid of nearly 100 robotics positioners able to place 7-fiber minibundles over a large field of view of $3.5 \mathrm{arcmin} \times 3.5 \mathrm{arcmin}$. The spectrograph is composed by a pseudo-slit, where the fibers are placed simulating a long slit; a slit shutter is placed just behind the pseudo-slit, a collimator, a $162 \mathrm{~mm}$ pupil where the volume phase holographic gratings are placed, and the camera with the detector. Here we describe the spectrograph optical rationale, the conceptual optical design and the expected system performance.
\end{abstract}

Keywords: : astronomical spectrographs, integral field units, multi-object fiber spectroscopy

\section{INTRODUCTION}

MEGARA stands for "Multi-Espectrógrafo en GTC de Alta Resolución para Astronomía" and meets the requirements of the Announcement of Opportunity for the new instrumentation for the Gran Telescopio Canarias, issued by GRANTECAN on September $14^{\text {th }}$, 2009. It is an instrument project lead by the Universidad Complutense Madrid (UCM) in partnership with the Instituto Nacional de Astrofísica, Óptica y Electrónica (INAOE), the Instituto de Astrofísica de Andalucía (IAA) and the Universidad Politécnica de Madrid (UPM). INAOE will develop this project in collaboration with the Centro de Investigaciones en Óptica (CIO). The scientific motivations of the MEGARA science team members can be grouped in two categories: (1) the study of Galactic and extragalactic nebulae and (2) the study of (or close to) point-sources with intermediate-to-high surface density. Among the former, our interests include the study of nearby galaxies or planetary nebulae and among the latter Galactic open stellar clusters, stellar populations in local group galaxies, intermediate-redshift dwarf and starburst galaxies and high redshift galaxy clusters are the main subject of our research activities. The fact that the MEGARA science team encompasses researchers with such a broad range of scientific interests also guarantees that the instrument will successfully serve to the much broader interest of the astronomical communities of the GTC consortium members. What is common to all our scientific interests is the need for an intermediate to high spectral resolution, in the range of $\mathrm{R} \approx 5600-17000$.

The instrument operates in three modes that cover different sky areas and that can run simultaneously: (1) the compact mode through a large central Integral Field Unit (IFU) with minimum fiber pitch, covering a field of view (FOV) on sky of $12 \times 14 \operatorname{arcsec}^{2}$; (2) the sparse mode with fibers covering $1 \times 1 \operatorname{arcmin}^{2}$ in three pointings and (3) the dispersed mode with a grid of nearly 100 robotics positioners able to place 7-fiber minibundles over a large field of view of $3.5 \mathrm{x}$

22nd Congress of the International Commission for Optics: Light for the Development of the World, edited by Ramón Rodríguez-Vera, Rufino Díaz-Uribe, Proc. of SPIE Vol. 8011, 80112D

2011 SPIE · CCC code: $0277-786$ X/11/\$18 - doi: 10.1117/12.903436

Proc. of SPIE Vol. 8011 80112D-1 
$3.5 \operatorname{arcmin}^{2}$ to carry out multi-object spectroscopy (MOS). All the fiber bundles are placed on the folded-Cassegrain focal station and are coupled to identical microlenses that convert the GTC $f / 17$ into the $f / 3$ beam needed for an optimum use of the fibers by minimizing the focal ratio degradation (FRD). All the bundles, one for the compact mode, one for the sparse mode and six for the dispersed mode, go to optically identical spectrographs placed on the Nasmyth platform. The spectrograph is composed by a pseudo-slit, where the fibers are placed simulating a long slit; a slit shutter is placed just behind the pseudo-slit, a collimator, a $162 \mathrm{~mm}$ pupil where the volume phase holographic (VPH) gratings are placed, and the camera with the detector. The optics manufacturing will be carried out at INAOE \& CIO. Here we present the high level requirements, the design rationality and the final optical conceptual design. This design is being revised as part of the Preliminary Design Phase.

\section{OPTICAL DESIGN}

\subsection{Design rationale}

The main characteristics of the spectrograph and the high level requirements are summarized in Table 1. With these requirements we developed the following design rationale.

Table 1. A subset of MEGARA main characteristics and spectrograph high level requirements (RQ).

\begin{tabular}{|ll|}
\hline Central IFU & $12 \times 14 \operatorname{arcsec}^{2}$ \\
IFU FOV & $1 \times 1 \operatorname{arcmin}^{2}$ \\
MOS (simultaneously with IFU) & 94 objects in $3.5 \times 3.5 \operatorname{arcmin}^{2}$ \\
\# of spectrographs & $8(7$ IFU + 1 MOS) \\
\# of spaxels / multiplexing & 5600 \\
GTC station & Folded-Cass (spectrographs@Nasmyth) \\
& \\
RQ-1. Wavelength range & $3700-9800 \AA$ \\
RQ-2 Spaxel size & 0.685 arcsec \\
RQ-3 Spectral resolution & $\mathrm{R}=5600-17000$ \\
RQ-4 Detector format & $4096 \times 4096$ \\
RQ-5 Pixel size & $15 \mu \mathrm{m} \times 15 \mu \mathrm{m}$ \\
RQ-6 Fiber core diameter & $100 \mu \mathrm{m}$ \\
RQ-7 Image quality & EED \\
RQ-8 Entrance $\mathrm{f} \mathrm{number} \mathrm{of} \mathrm{the} \mathrm{resolution} \mathrm{element} \mathrm{(4pix)}$ \\
RQ-9 Space between two adjacent fibres & $\mathrm{f} / 3$ \\
\end{tabular}

Plate scale: the plate scale sets the fcoll/fcam ratio. As 0.685 " has selected on the sky and the spectral element is

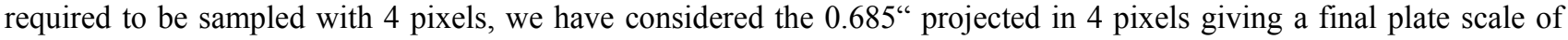
$0.171 \mathrm{arcsec} /$ pixel on the detector.

Scale reduction factor: we will project the $100 \mu \mathrm{m}$ fiber size core in 3.33 pixels by design. As the pixel size is $15 \mu \mathrm{m}$, 3.3 pixels are $50 \mu \mathrm{m}$, what implies a scale reduction between the telescope focal plane and the detector of a factor of 2 . This factor will fix the ratio between the f-numbers of collimator and camera.

Collimator f-number: the spectrograph entrance f-number is chosen to minimize the FDR of the fiber link, thus $f / 3$ is used at the entrance and exit of the fiber becoming the collimator f-number.

Camera f-number: since the ratio between the f-number of the collimator and the f-number of the camera is 2 and the $f$ number of collimator is 3 then the f-number of the camera 1.5 what is feasible. Faster (smaller) $\mathrm{f}$-numbers for the camera become very difficult for such a large and complex camera.

Pupil size: the FOV will limit the minimum pupil size. As we move to wider FOV the total focal length of the collimator and camera has to be increased in order to maintain the image quality. With the current experience filling $2 \mathrm{~K}$ pixels in the spatial direction will require a minimum pupil size of $80 \mathrm{~mm}$ and filling $4 \mathrm{~K}$ pixels in spatial direction will require a minimum of $160 \mathrm{~mm}$ pupil in order to manage field aberrations. This pupil size has been taken as reference for this 
design and this will limit the maximum FOV at the entrance of the spectrograph (pseudo-slit). The current design has a $162 \mathrm{~mm}$ pupil diameter.

Spectral resolution: the linear spectral resolution is proportional to the camera focal length. Thus, using a smaller pupil will shorten the focal length and the linear dispersion at the end. For example, using a half diameter pupil would decrease the resolution to half of the value if we maintain the four pixels per resolution element. In order to recover the nominal resolution two options have been explored in the feasibility study but only one is valid at expense of FOV coverage. The first one is doubling the angular dispersion of the dispersion element. This can be done at $\mathrm{R}=5000$ but not at high resolution (HR) $\mathrm{R}=17000$ as we are in the limit of the geometry, so it is not an option if we are required to maintain HR. The second option was decreasing the fiber size to half of the value (thus $50 \mu \mathrm{m}$ ) and using two pixels per resolution element but it was not acceptable due to the scientific requirements. Therefore as far as we have discarded these two solutions, we have used a pupil as large as $162 \mathrm{~mm}$.

\section{Spectral resolution and geometry:}

$$
\frac{d \beta}{d \lambda}=\frac{m n}{\operatorname{COS} \beta}
$$

The grating equation (above) for the angular dispersion sets the geometry of the light angle of incidence (AOI) on the grating, $\lambda$ is the wavelength; $\beta$ is the AOI, $\mathrm{n}$ the number of lines $/ \mathrm{mm}$ and $\mathrm{m}$ the diffraction order. The best VPH performance regarding efficiency is normally obtained at first order $\mathrm{m}=1$.

This means that setting a HR mode will require a high AOI on the grating, while a low resolution (LR) mode requires a low AOI on the grating. An example is shown in Fig. 1

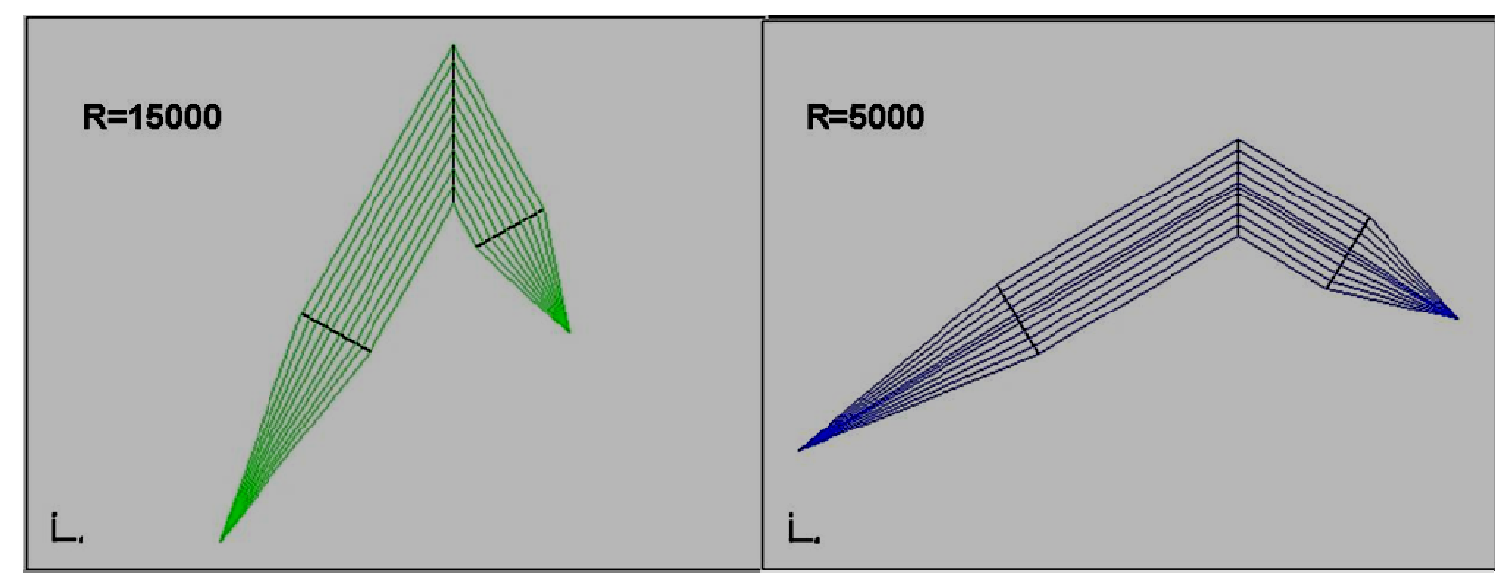

Figure 1. Example to illustrate the different angle on the grating needed for two extreme spectral resolutions with values of the resolving power, $\mathrm{R}$ of 15000 (left) and 5000 (right), respectively.

Different solutions can be offered for fitting a wide range of spectral resolutions: a) to consider two spectrographs geometries, everything is the same except the envelope and the fixed geometry. This means to have two different spectrographs, one specific for HR and the other one for LR. This is optimum in terms of optical design; b) a single spectrograph with an articulated camera-grating that changes the geometry. In this case, the instrument would require a higher envelope and motorization of the grating and the camera-detector stage, what is not desirable; c) a single spectrograph with a fixed geometry between the HR and the LR optimum angles. The final geometry on the grating is obtained by sandwiching the gratings between two prisms, what will change the beam's angle to the required one. 
All these previous options share the same optical design regarding the collimator and camera design. However, some differences are found regarding the gratings, and we have analyzed them in order to arrive to the current configuration leading us to conclude that option (c) is the best one but even in this case we have to propose a novel design for the highest resolution mode based on segmented pupil gratings discussed in [1].

Spectral elements: the VPHs are proposed as the spectral dispersers of the instrument. Ruled gratings are not available with the number of lines/mm required while surface holographic gratings are not so efficient. Moreover, transmission gratings are preferred over reflective ones because of the smaller size of the camera optics, as because in this case the camera elements can be located very close to the dispersive element.

In the VPHs, the index pattern (hologram) is photo-recorded in a dichromate gelatin. The hologram parameters, lines $/ \mathrm{mm}$ and AOI on the grating, give the wavelength that meets the Bragg condition. These values provide the required resolutions for the proposed gratings. The hologram thickness can be tuned to avoid a high dependence in the efficiency when out of the Littrow angle [2].

The angle of incidence within the gelatin is given by the standard grating equation. Where $m=1$ is the order, $\lambda$ is the Bragg wavelength, $d$ is the grating spacing, and $n=1.27$ is the gelatin index:

$$
2 d n \operatorname{SIN} \beta_{m}=m \lambda
$$

The overall geometry implies that the required working angles will be around $26^{\circ}$ in the LR and around $70^{\circ}$ in the HR. In order to change the AOI from a fixed geometry to the different angles required, prisms are used, the grating will be sandwiched inside. The prism material will depend on the grating AOI. When the light goes from the gelatin to the material substrate or vice versa, we apply the Snell law:

$$
n_{\text {gelatine }} \operatorname{SIN} \beta=n_{\text {prism }} \operatorname{SIN} \alpha
$$

Spectral resolution and fiber size: there is another way of changing the spectral resolution and this is by using different fiber sizes for different resolutions. For example if we use $100 \mu \mathrm{m}$ core fibers for $\mathrm{R}=10000$ (projecting 1 fiber on 4 pixels) and $50 \mu \mathrm{m}$ for $\mathrm{R}=20000$ (projecting 1 fiber on 2 pixels) we could have both resolutions with the same diffraction element. However, we have discarded this solution since of the most important scientific requirements is the use of the same type of fibers to have the same spaxel on sky with all the resolutions. Moreover, the plate scale on the sky covered by a $50 \mu \mathrm{m}$ fiber is very small $(0.3 \mathrm{arcsec})$ what implying a sub-seeing sampling and a much lower sky coverage, which is one of the most important scientific requirement.

\subsection{Final spectrograph conceptual design}

After studying different configurations during the Feasibility Study we decided to choose a fully refractive system for the spectrograph. The spectrograph is composed by a pseudo-slit, where the fibers are placed simulating a long slit. A slit shutter is placed just behind the pseudo-slit. A collimator is composed by five lenses: one singlet and two doublets being these two doublets mounted on a linear stage that will be the focusing mechanism. The pupil has $162 \mathrm{~mm}$ diameter and it is the location for the VPH-gratings. In the current conceptual design we propose to mount the order sorting filter when needed together with the grating in the opto-mechanical mount. Once the beam passes through the grating it goes to the camera, composed by two doublets and three singlets, and then to the detector. We are considering that the last lens is also the cryostat window. The MEGARA team has experience with this configuration that offers the advantage of increasing the throughput by saving two surfaces. The main properties of the different spectrograph subsystems with optical functionality are the followings:

Pseudo-Slit: the length of the slit is $122 \mathrm{~mm}$. The fiber pseudo-slit is curved on a sphere surface of radius of curvature of $500 \mathrm{~mm}$. Several ways of doing the pseudo-slit have been discussed with the potential providers and the final pitch will be the one more similar to the requirement $(178 \mu \mathrm{m})$. The current baseline could imply a reduction of this value up to $170 \mu \mathrm{m}$, coincident with the fiber mechanical coating, what is acceptable. The final number of fibers in the pseudo-slit will be decided in the next phase but we will have a number between 682 and 717 fibers.

Collimator: the collimator is composed by a singlet and two doublets as seen in Fig 2 with a focal length of $495 \mathrm{~mm}$. It is a f-number 3 refractive system. 


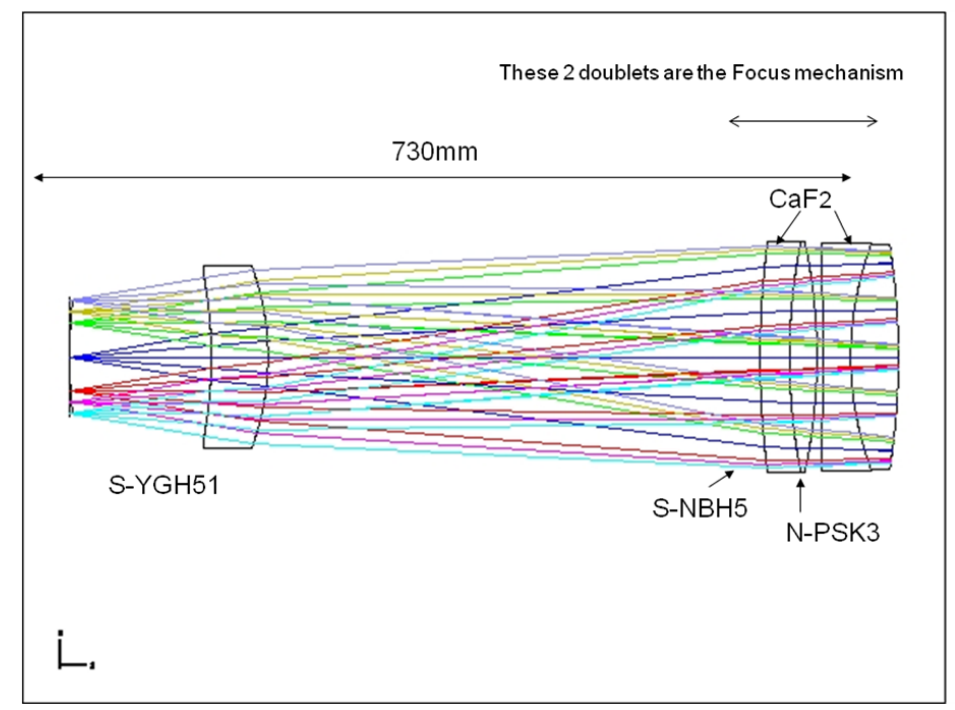

Figure 2. Collimator layout. It is composed by one S-YGH51 singlet and two doublets, each of them with one CaF2 lens.

Table 2. Collimator lenses parameters. L2 - L3 and L4 -L5 are cemented doublets.

\begin{tabular}{|c|c|c|c|c|c|}
\hline Element & Material & $\mathrm{R}_{1}(\mathrm{~mm})$ & $\mathrm{R}_{2}(\mathrm{~mm})$ & Central thickness $(\mathrm{mm})$ & Clear aperture $(\mathrm{mm})$ \\
\hline L1 & S-YGH51 & -386.9 & -233.7 & 50.0 & 152.0 \\
\hline L2* & S-NBH5 & 794.9 & 528.6 & 26.0 & 198.0 \\
\hline L3* & $\mathrm{CaF}_{2}$ & 528.6 & -488.6 & 24.0 & 198.0 \\
\hline L4* & N-PSK3 & -7752.7 & 287.2 & 25.0 & 193.0 \\
\hline L5 $*$ & $\mathrm{CaF}_{2}$ & 287.2 & -646.2 & 42.0 & 190.0 \\
\hline
\end{tabular}

Pupil: the pupil size is $162 \mathrm{~mm}$ and the gratings will be located in this position.

Geometry: fixed at $68^{\circ}$ between collimator and camera.

Camera: the camera is composed by two doublets and 3 singlets as seen in Figure 3 . The focal length is $243 \mathrm{~mm}$. It is a refractive design with a f-number 1.5 . The image field is $61.4 \mathrm{~mm} \times 61.4 \mathrm{~mm}$ covering $4 \mathrm{~K}$ x $4 \mathrm{~K}$ pixels. The characteristics of the camera lenses are summarized in table 3. The total expected transmission of the optical system, excluding the pupil elements, is shown in Figure 4. It was calculated assuming antireflection coatings at the interfaces glass/air of $1.5 \%$, perfect interfaces between glasses and considering the internal transmission of the glasses provided by Zemax.

Pupil elements: different types of pupil elements, all of them based on VPH-type gratings, can be accommodated in the pupil position to provide the different spectral modes, with resolution power ranging between 5600 and 17000 . LR units can be built with simple gratings sandwiched between two flat windows; mid units are provided with the gratings sandwiched between two symmetric prisms that allow the beam to incident on the VPH. However, for the HR mode the prisms would be so heavy and thick that would make them inefficient. For this reason and in order to provide the wide resolution range required we developed the concept of a sliced pupil grating, the details are discussed in [1]. Figures 5 and 6 show different configurations for LR, mid-resolution (MR) and HR. 


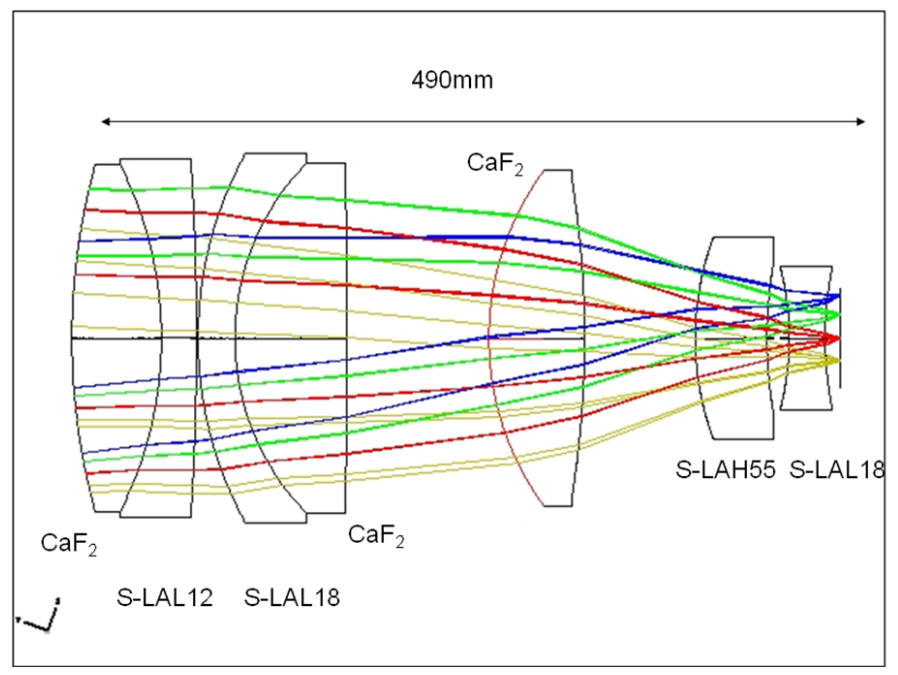

Figure 3. Camera layout. It is composed by two doublets, each of them with one CaF2 lens, and three singlets.

Table 2. Camera lens parameters. L1 - L2 and L3 - L4 are cemented doublets.

\begin{tabular}{|c|c|c|c|c|c|}
\hline & Material & $\mathrm{R}_{1}(\mathrm{~mm})$ & $\mathrm{R}_{2}(\mathrm{~mm})$ & $\begin{array}{c}\text { Central thickness } \\
(\mathrm{mm})\end{array}$ & $\begin{array}{c}\text { Clear aperture } \\
(\mathrm{mm})\end{array}$ \\
\hline L1* $^{*}$ & $\mathrm{CaF}_{2}$ & 408.4 & -240.5 & 56.2 & 40.0 \\
\hline L2* $^{*}$ & S-LAL12 & -240.5 & -1792.9 & 22.0 & 240.0 \\
\hline L3* & S-LAL18 & 239.6 & 156.7 & 23.2 & 258.0 \\
\hline L4* & $\mathrm{CaF}_{2}$ & 156.7 & -8941.5 & 70.0 & 240.0 \\
\hline L5 & $\mathrm{CaF}_{2}$ & 176.7 & -617.5 & 60.0 & 230.0 \\
\hline L6 & S-LAH55 & 181.5 & 272.7 & 45.0 & 130.0 \\
\hline L7 & S-LAL18 & -180.7 & 224.1 & 22.6 & 90.0 \\
\hline
\end{tabular}

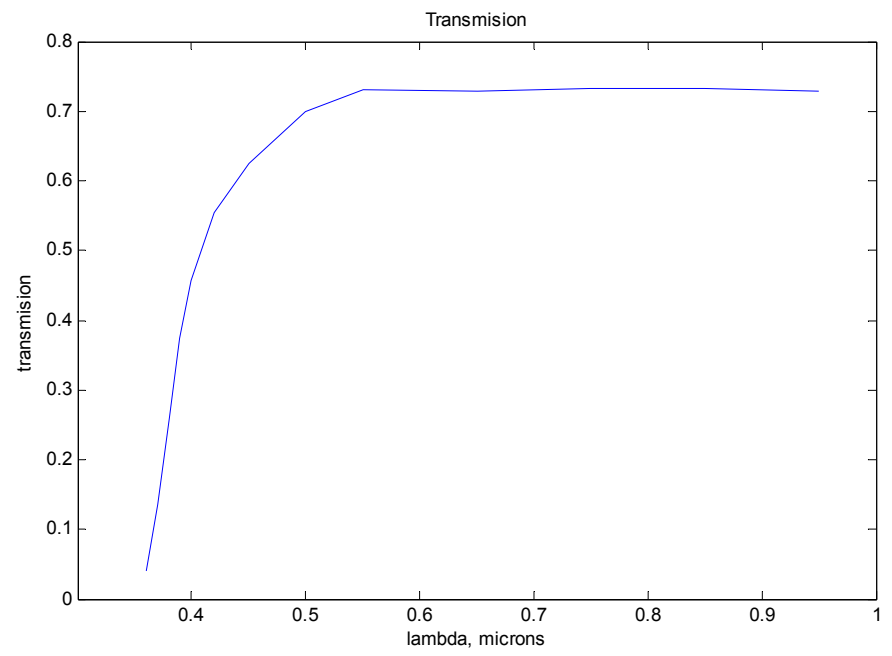

Figure 4. Predicted transmission of MEGARA's collimator and camera. The assumptions are explained in the text. 


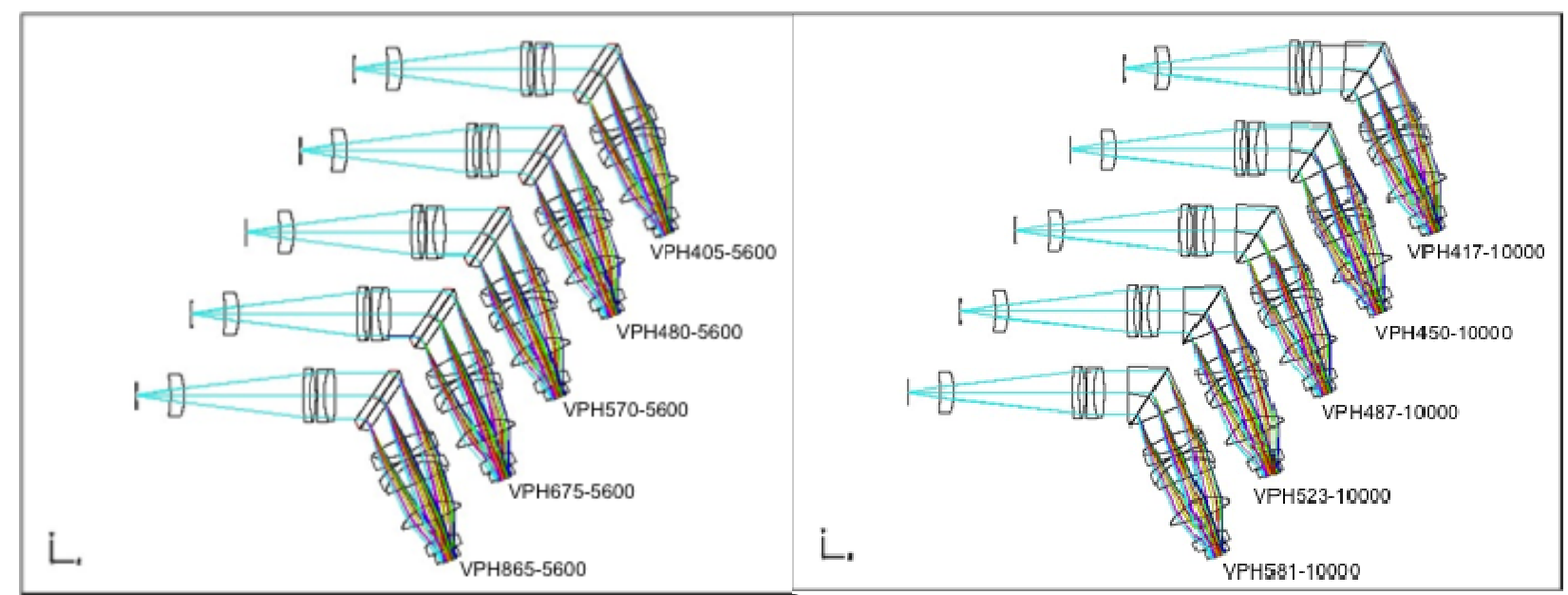

Figure 5. Left: MEGARA configuration in the low resolution mode $(\mathrm{R}=5600)$ where the pupil element is a VPH grating sandwiched between two windows. Right: MEGARA configuration in the mid resolution mode $(\mathrm{R}=10000)$ where the pupil element is a VPH grating sandwiched between two prisms to compensate the beam angle.

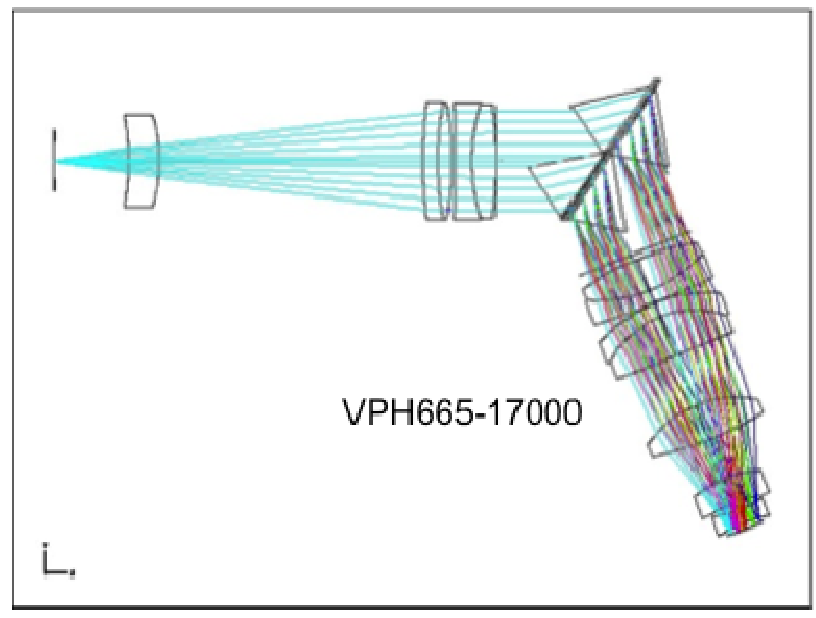

Figure 6. Design of the $\mathrm{R}=17000$ grating centered at 6500 A with a pupil segmented in 2 slices [1].

\subsection{Image quality performance}

The image quality requirement is to have the resolution element in four pixels i.e. $60 \mu \mathrm{m}$. The value that contains the $80 \%$ of the encircled energy coming from a fiber whose projection is $50 \mu \mathrm{m}$ is a diameter of $35 \mu \mathrm{m}$, as shown in Fig. 7 . Thus,

$$
\mathrm{EE}_{80}=60^{2}-35^{2}=48.7^{2}
$$

For this reason the total value of the $\mathrm{EE}_{80}$ through the optical system has to be lower or equal to $48.7 \mu \mathrm{m}$ in diameters, or $24.36 \mu \mathrm{m}$ (half of $48.7 \mu \mathrm{m}$ ) in radius. From the previous calculations the value of the requirement when analyzing the image performance will have a value of $\mathrm{EER}_{80} \leq 24.4 \mu \mathrm{m}$. 


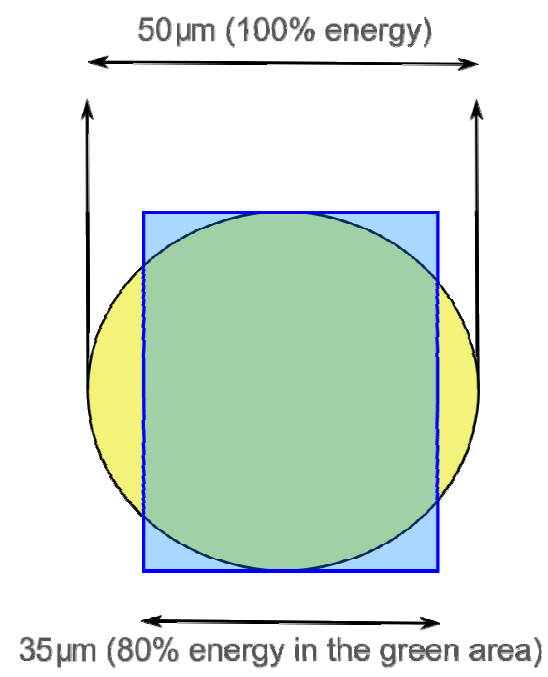

Figure 7. The value of the diameter of a circle that contains the $80 \%$ of the energy from a $100 \mu \mathrm{m}$ fiber, whose circular projection on the detector is a circle of a diameter of $50 \mu \mathrm{m}$, is $35 \mu \mathrm{m}$.

We analyzed the image quality performance obtained with the different configurations of the instrument i.e. for different spectral resolutions. For each grating we obtained the projection of the spectra and the spot diagram at different fiber positions on the pseudo-slit, covering the whole pseudo-slit length and therefore the whole range of AOI on the grating. Additionally, a total of six wavelengths were analyzed in each configuration providing a graph where the values of $\mathrm{EER}_{80}$ is plotted as a function of the position. An example of the plots obtained for each configuration is shown in figure 8 and 9 for a $\mathrm{R}=5600$ and $\lambda \mathrm{c}=570 \mathrm{~nm}$. All the configurations are within requirements and with a good margin for EB.

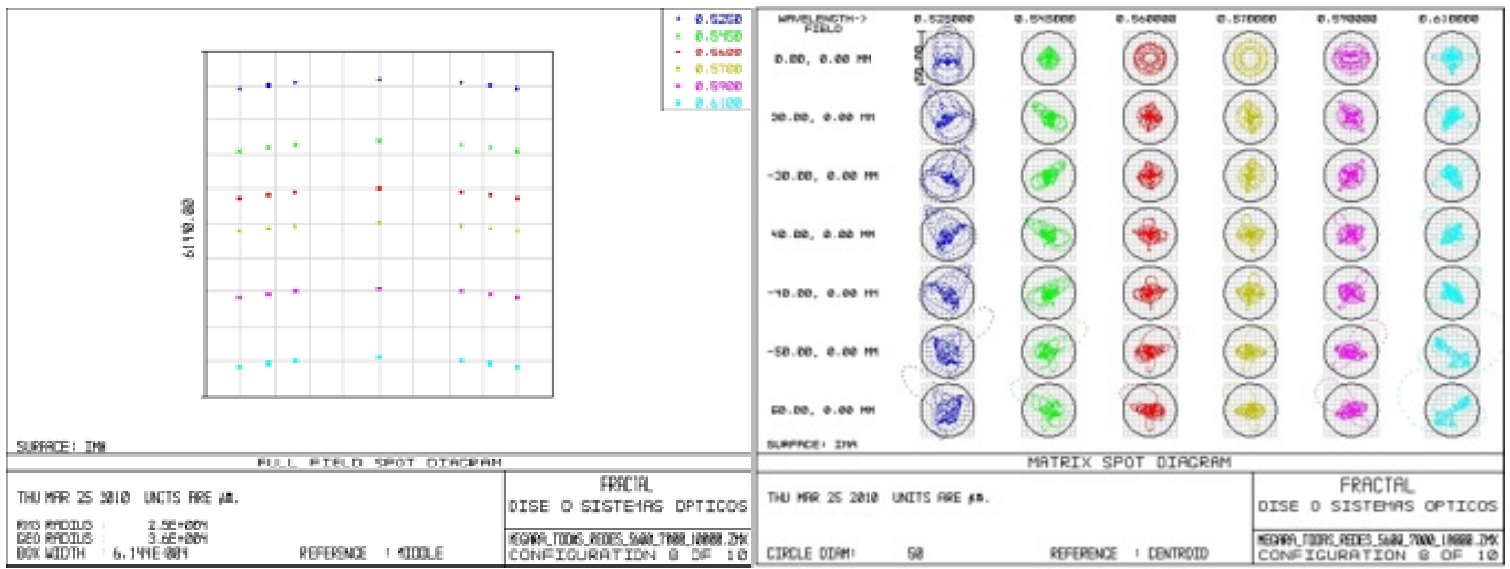

Figure 8. Left: projection on the detector of the spectra from fibers at different positions of the pseudo-slit in the configuration of the gratingVPH570-5600. Right: spot diagram of the spectra from fibers at different positions of the pseudo-slit in the configuration of the same grating. Different colors correspond to different wavelengths. 

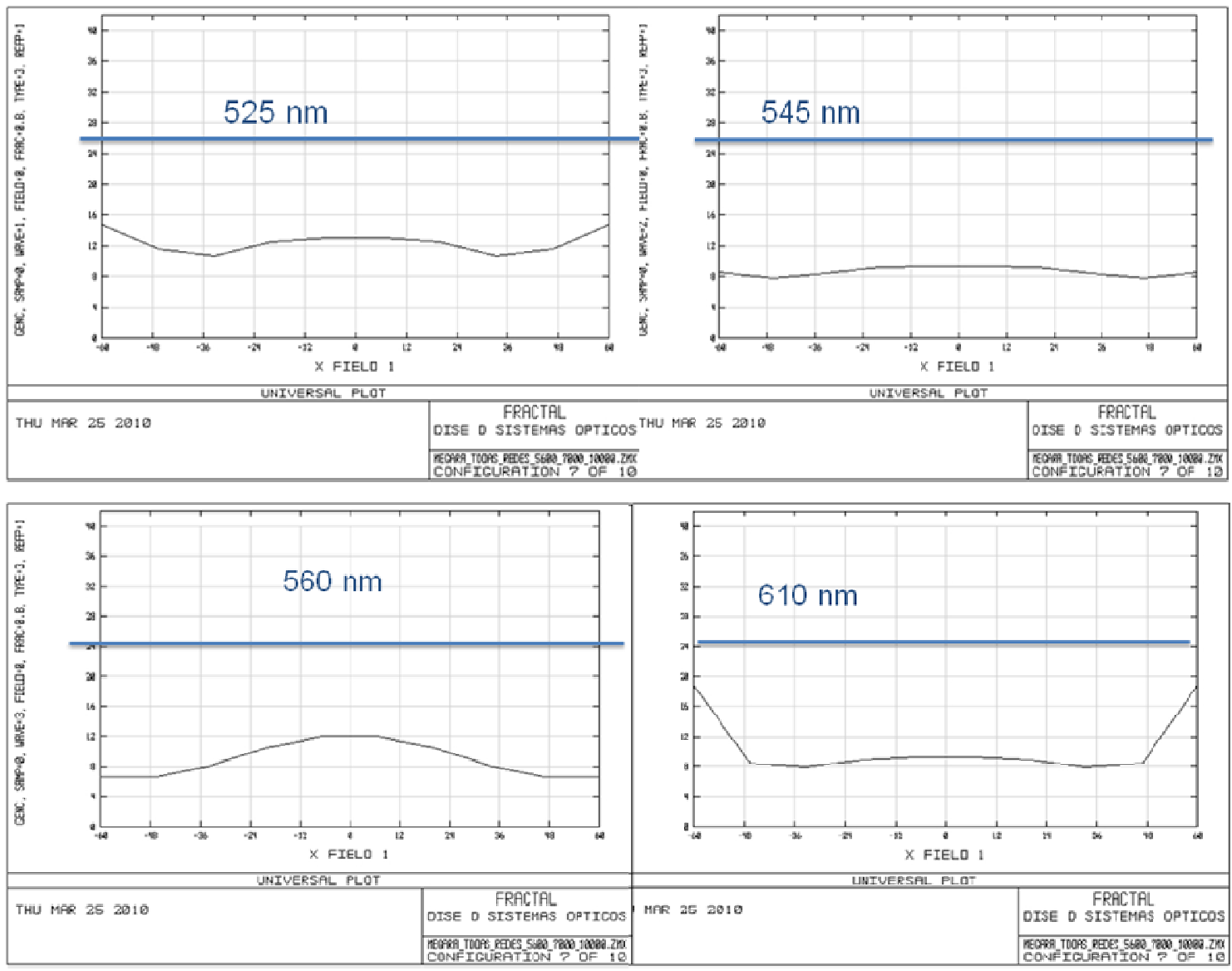

Figure 9. Four examples of the $\mathrm{EER}_{80}$ plots as a function of the position of the FOV (on the pseudo-slit) with the grating VPH570-5600, for the wavelengths indicated. The horizontal line represents the image quality requirement. For each configuration we obtained this kind of plots for six wavelengths, all the fields and wavelengths are within requirements.

\subsection{Image Quality Error Budget}

For optical analysis the evaluation of the EB will not be made in terms of $\mathrm{EED}_{80}$, but in terms of RMS spot radius, and considering 1d Gaussian profile in the spectral direction. Thus, in order to compute the $\mathrm{EB}$, we will translate the $\mathrm{EE}_{80}$ to the $\sigma$ of a Gaussian. The $80 \%$ of the energy under a $1 \mathrm{~d}$ Gaussian is contained under $\pm 1.28 \sigma$.

Thus $\mathrm{EED}_{80}=48.7 \mu \mathrm{m}$, will become $\mathrm{EER}_{80}=24.3 \mu \mathrm{m}$ or

$$
\sigma=\mathrm{EER}_{80} / 1.28=19 \mu \mathrm{m}
$$

where $19 \mu \mathrm{m}=\sigma$ associated to $80 \%$ of EER. The error budget is built as:

$$
\sigma_{\text {total }}^{2}=\sigma_{\text {design }}^{2}+\sigma_{\text {fabrication }}^{2}+\sigma_{\text {alignment }}^{2}+\sigma_{\text {thermal }}^{2}+\ldots
$$

Table 4 shows the estimated values for the different image quality error budget contributors including the total error budget constructed as shown above. Given the estimated values it is expected and achievable to be below the requirement. Regarding the optics manufacturing we will carry out an extensive tolerances analysis for each construction parameter of the optical system using different methods to optimize the manufacturing process of each surface. One of the advantages of having the optics fabricator -in this case INAOE \& CIO- as part of MEGARA team is 
that we will be able to iterate with the optical designer to achieve the finest equilibrium between an excellent performance and optimum manufacturing process.

Table 4. Estimated values for the different image quality error budget contributors.

\begin{tabular}{|l|c|l|}
\hline ITEM & $\begin{array}{c}\sigma \\
(\mu \mathrm{m})\end{array}$ & Comment \\
\hline Nominal performance & 9 & Nominal design in one representative mode \\
\hline $\begin{array}{l}\text { Fabrication (lens thickness, wedge, } \\
\text { surface irregularity, curvature) }\end{array}$ & 10 & $\begin{array}{l}\text { Will be evaluated with Monte Carlo runs in } \\
\text { normal distribution }\end{array}$ \\
\hline $\begin{array}{l}\text { Assembly and alignment (axial and } \\
\text { lateral decentration, tilts). }\end{array}$ & 9.1 & $\begin{array}{l}\text { Will be evaluated with MC. Integration } \\
\text { compensators are expected in the camera }\end{array}$ \\
\hline Thermal & 5 & $\begin{array}{l}\text { Operation temperature ranges shall be } \\
\text { introduced }\end{array}$ \\
\hline $\begin{array}{l}\text { Glass homogeneity and melt index } \\
\text { tolerance }\end{array}$ & 5 & Analytical model \\
\hline Marging for the EB & 5 & \\
\hline $\begin{array}{l}\text { TOTAL (rms squared) } \\
\text { Requirement }<19 \mu \mathrm{m}\end{array}$ & 18.4 & \\
\hline
\end{tabular}

\section{CONCLUSIONS}

We developed an optical design that fulfills all the scientific requirements. The design is consistent with an $\mathrm{f} / 3$ fiber fed spectrograph, projected onto a $4 \mathrm{k} \mathrm{x} 4 \mathrm{k}$ detector and with a resolution element of 4 pixels. The design is based on a collimator-camera design with a fixed angle between two elements, what has been chosen to allow the wide spectral resolution range required in the scientific requirements. The presented design covers a range between $\mathrm{R}=5600$ and 17000 what has been optimized according to: (a) GTC requirements, (b) the science team requirements and (c) the need of using a single detector in each spectrograph, what has been considered a design requirement.

Spectral resolution is done through VPH gratings. These VPH gratings will be sandwiched between two flat windows (in the $\mathrm{LR}$ mode at $\mathrm{R}=5600$ ) and two prisms (in the MR mode at $\mathrm{R}=10.000$ ). In the case of the HR mode our baseline is to use a sandwich between two prisms each side, using our novel design of sliced pupil gratings [1].

All the modes have room enough in the Image Quality Error Budget to assure that the high level scientific requirements will be fulfilled. Evaluation has been done for all VPHs defined by the scientific requirements and along the whole wavelength range and spatial distribution along the pseudo-slit. Finally, all blanks have quite standard sizes. We will review all materials in the Preliminary Design Phase to assure blanks availability and to improve the performance.

\section{REFERENCES}

1. Sánchez-Blanco, E., García-Vargas M., Maldonado, M., Gallego, J., Gil-de-Paz, A., Carrasco, E., Pérez, A., Martínez-Delgado, I \& Zamorano J., "Sliced-pupil grating: a novel concept for increasing spectral resolution", this proceddings.

2. Barden, S.C, Arns, J.A., Colburn, W.S. \& Williams, J.B. , " Volume-phase holographic gratings and the efficiency of three simple VPH gratings", PASP, 112, 809, 2000 